\title{
Effects of Empathic Ability and Campus Life Stress on Stress Coping Behaviors in Dental Hygiene Students
}

\author{
Ji-Hyoung Han, Jin-Young Yang ${ }^{1}$, and Ji-Min Hwang ${ }^{2, \dagger}$ \\ Department of Dental Hygiene, Suwon Science College, Hwaseong 18516, \\ ${ }^{1}$ Department of Dental Hygiene, Daejeon Institute of Science and Technology, Daejeon 35408, \\ ${ }^{2}$ Department of Dental Hygiene, Baekseok Culture University, Cheonan 31065, Korea
}

\begin{abstract}
The purpose of this study was to investigate the effect of empathic ability and campus life stress on the stress coping behaviors among 395 dental hygiene students. The analysis was performed using PASW Statistics ver. 18.0, and the following conclusions were obtained. The empathic concern factor was the highest in the subscale of empathic ability (3.60). The interpersonal relationship stress was 1.65 and the task-related stress was 2.72. The stress coping behaviors were the highest among the sub-domains, with 3.69 for wishful thinking. The differences of stress coping behaviors according to general characteristics were as follows. The lower the age and grade, the higher the problem-focused coping; when they were religious there was a high pursuit of seeking social support. When they were satisfied with their economic level, wishful thinking was high. There was a low negative correlation between empathic concern and interpersonal relationship stress in campus life $(p<0.01)$. There was a positive correlation between personal distress and task-related stress $(p<0.001)$. The relationship between empathic ability and stress coping behaviors was most associated with personal distress and wishful thinking. Among the sub-domains of stress coping behaviors, factors that have a common impact on personal distress and seeking social support are viewpoint acceptance. Factors supporting emotional focus and wishful thinking were task-related stress. Dental hygiene students are not able to completely eliminate the stress that they are actually under. However, as the research results show, it is necessary to use stress coping techniques to cope effectively with individual tendencies and situations, and to improve the ability to sympathize with another individual.
\end{abstract}

Key Words: Campus life stress, Empathic ability, Stress coping behavior

\section{Introduction}

The word stress was derived from the Latin word stringere, which was used in 19th century physics to mean 'holding something tight,' and was used in the same sense, as it is now used, in 20th century medicine ${ }^{1)}$. Stress is a state of psychological and physical tension that individuals feel when they are faced with a difficult environment or condition ${ }^{2}$. Everyone experiences stress due to complicated social structure, excessive work and study, and interpersonal relationships. In recent years, attention has been focused on the stress coping process from the cognitive-phenomenological standpoint, unlike in the past where attention was paid to structural factors such as situational variables and personal characteristics ${ }^{3)}$. Stress is not a direct problem in itself, but rather has adverse effects due to improper coping with the stress ${ }^{4)}$. Individuals who experience various stressors may perceive stress differently according to individual perception and psychological reaction; there is a great difference among individuals. In the transactional model of stress, more emphasis is placed on coping with stress rather than on stress itself, and related systematic studies have been conducted. In terms of stress coping strategies, pro- 
blem-focused coping and seeking social support can be classified as active stress coping, whereas emotional relief coping and wishful thinking coping can be classified as passive stress coping ${ }^{3)}$. Stress coping can also be largely divided into two coping strategies: problem-focused coping aimed at changing the stressful situation, and emotion-focused coping that addresses the subjective elements of emotions associated with stress. When some constructive measures and controls are perceived to be feasible, problem-focused coping is high. When it is perceived to be a difficult-to-control situation, emotion-focused coping often occurs 5 ).

In addition, the word empathy, derived from German Einfhlung at the end of 19th century, was translated into the Greek empatheia, and is now used as empathy. Empathy is a synonym for 'en' meaning 'inside' and 'pathos' meaning 'suffering' or 'emotion,' and means to limitedly feel, communicate, and recognize the feelings, thoughts, and psychological state from the other's perspective $^{6}$. The reason why such empathic understanding is important is because empathy helps to recognize the root cause of conflicts within a group, provide opportunities to solve group conflicts, promote positive interpersonal relationships, and act as a factor to facilitate individual growth such as academic performance and increased capacity for coping ${ }^{7)}$.

College students in late adolescence have a level of self-identity development higher than in individuals in middle and high school ${ }^{8)}$, and they experience various forms of stress as they adapt to changes in self-regulation and independent socio-psychological living environments ${ }^{9)}$. The stress experienced by college students can be of many forms, including academic performance, career, employment, and interpersonal relationship stress. When stress becomes gradually chronic, symptoms associated with various physical and mental pathologies such as physiological, behavioral, and emotional symptoms may occur $^{10)}$. The difficulties caused by the stress of campus life can extend beyond problems related to academic performance, employment, and interpersonal relationships to addiction, mental suffering such as depression, various crimes and suicide ${ }^{9)}$. Therefore, properly coping with stress arising in the surrounding environment, balancing harmoniously with the environment, and maturing are very important tasks, not only for college students as future members of society, but also for the society. In particular, dental hygiene students, as compared to other departments, experience a lot of stress due to the rigorous curriculum, excessive academic workload, clinical practice, and psychological pressure to pass the national examination.

The present study aimed to investigate the effects of empathic ability and campus life stress on stress coping behaviors in dental hygiene students, and to provide baseline data that can contribute to the development of intervention programs and holistic educational strategies for dental hygiene students' healthy and smooth campus life, and increase their coping ability.

\section{Materials and Methods}

\section{Subjects and methods}

The subjects of this study were second and third year dental hygiene students, randomly selected from 1 college located in Gyeonggi-do, 1 college in Chungcheongnam-do, and 1 college in Daejeon Metropolitan City, South Korea. The survey was conducted from April 1, 2017 to April 20, 2017. The purpose of the study was explained and those who agreed to participate signed an informed consent. The subjects then completed a self-report questionnaire. The sample size was calculated with a significance level of 0.05 , a statistical power of 0.8 and an effect size of 0.3 using G Power 3.1 software program. As a result, a total of 297 subjects were required for correlation and multiple regression analysis; the data obtained from 395 subjects were analyzed. The present study was approved by the institutional review board at Daejeon Institute of Science and Technology (IRB no. 2017-003-015).

\section{Instruments}

The general characteristics of the subjects that were investigated totaled 8 items including sex, age, school year, religion, self-perceived health status, satisfaction with economic level, college life, and with the dental hygiene major. The self-perceived health status, satis- 
faction with economic level, campus life, and with the dental hygiene major were scored on a 5-point Likert scale. Then, 4 and 5 points were adjusted to be 'good' and 'satisfied,' respectively; 3 points were adjusted to be 'normal'; and 1 and 2 points were adjusted to be 'bad' and 'unsatisfied,' respectively. Emphatic ability was measured using the Korean version of the interpersonal reactivity index originally developed by Davis ${ }^{1)}$ that was translated and adapted by Kang et al. ${ }^{11)}$. This instrument consists of 4 subdomains with a total of 28 items, including 7 items on perspective taking, 7 items on fantasy, 7 items on empathic concern, and 7 items on personal distress. Each item was scored on a 5-point Likert scale, and a higher score indicated a higher emphatic ability. The reliability of this instrument was Cronbach's $=0.776$.

Campus life stress was measured by using the life stress scale for students developed by Chon et al. ${ }^{12)}$, and was classified into interpersonal relationship stress and task-related stress domains. The scale consists of a total of 50 items on the interpersonal relationship stress and the task-related stress domains. The subdomains of the interpersonal relationship stress domain include relationship with friends of the same sex (5 items), friends of the opposite sex (6 items), family (6 items), and faculty (6 items). The subdomains of the task-related stress domain include problems related to academic grade (7 items), economy (7 items), future ( 8 items) and value (5 items). Each item was scored on a 5-point scale, and a higher score indicated more stress. The reliability was Cronbach's $=0.930$.

The ways of stress coping were measured using a scale that was developed through factor analysis by $\mathrm{Kim}^{13)}$ based on the Ways of Coping Checklist developed by Folkman and Lazarus ${ }^{14)}$. The subdomains consist of a total of 24 items on problem-focused coping (6 items), seeking social support (6 items), emotional relief coping (6 items) and wishful thinking coping (6 items). A higher score on a 5-point Likert scale indicated better coping with stress. The reliability was Cronbach's=0.798.

\section{Data analysis}

The data were analyzed using PASW Statistics ver. 18.0 (IBM Co., Armonk, NY, USA). General characteristics, emphatic ability, campus life stress, and stress coping behaviors were calculated and presented as frequency, percentage, mean and standard deviation. A t-test and one-way ANOVA were performed to compare differences in stress coping behaviors according to the general characteristics. Homogeneity of variance test was performed using Levene's test, and Scheffe's post-hoc test was performed to identify significant difference between the groups. Pearson's correlation analysis was used to examine the relationship between empathy ability, campus life stress, and stress coping behaviors. Stepwise multiple regression analysis was performed to examine factors affecting stress coping behaviors in the subjects. The significance level was set at 0.05 .

\section{Results}

\section{Empathic ability, campus life stress, and stress} coping behavior in the subjects

Empathic ability, campus life stress, and stress coping behaviors were analyzed using descriptive analysis. As a

Table 1. Level of Empathic Ability, Campus Life Stress and Stress Coping Behaviors $(n=395)$

\begin{tabular}{lc}
\hline \multicolumn{1}{c}{ Variable } & Value \\
\hline Empathic ability & $3.19 \pm 0.464$ \\
Perspective-taking & $3.53 \pm 0.652$ \\
Fantasy scale & $3.60 \pm 0.524$ \\
Empathic concern & $3.28 \pm 0.485$ \\
Personal distress & \\
Campus life stress & $1.65 \pm 0.573$ \\
Interpersonal relationship stress & $1.44 \pm 0.684$ \\
Homogeneity friend & $1.58 \pm 0.777$ \\
Boy or girl friend & $1.73 \pm 0.486$ \\
Domestic relations & $1.84 \pm 0.873$ \\
Professor relations & $2.72 \pm 0.644$ \\
Task-related stress & $3.38 \pm 0.730$ \\
Academic problem & $2.37 \pm 0.986$ \\
Economic problem & $2.57 \pm 0.795$ \\
Future problem & $2.57 \pm 0.986$ \\
Values problem & \\
Stress coping behaviors & $3.06 \pm 0.633$ \\
Problem focus coping & $3.28 \pm 0.681$ \\
Seeking social support & $2.96 \pm 0.606$ \\
Support emotional focus & $3.69 \pm 0.611$ \\
Wishful thinking &
\end{tabular}

Values are presented as mean \pm standard deviation. 


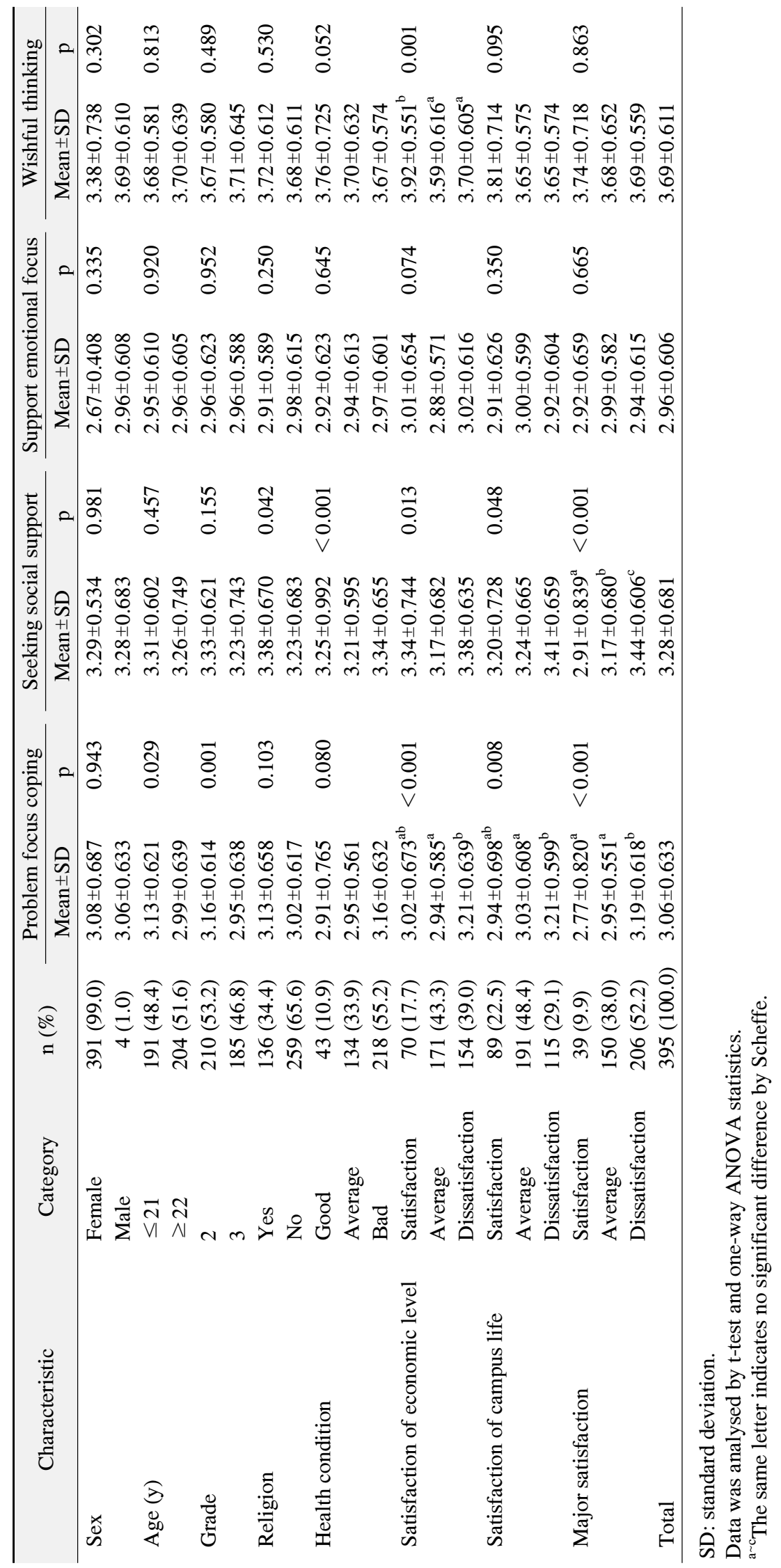


result, the score for empathic concern factor was the highest with 3.60 points among the subdomains of empathic ability. For campus life stress, the score for interpersonal relationship stress of 1.65 points was lower than the task-related stress of 2.72 points. The score for faculty relationship stress was the highest (1.84) among the subdomains of interpersonal relationship stress, while the score for grade stress (3.38) was the highest among the subdomains of task-related stress (Table 1). The score for wishful thinking coping was the highest with 3.69 points among the subdomains of stress coping behaviors, and the score for emotional relief coping was the lowest with 2.96 points.

\section{Difference in stress coping behaviors according to general characteristics}

Differences in stress coping behaviors according to the general characteristics of the subjects are shown in Table 2. By sex, 99.0\% $(n=391)$ were female and $1.0 \% \quad(n=4)$ were male. As age and college year were lower, coping via problem-solving was higher $(p<0.05)$. If the subjects were religious, seeking social support coping was high. If the subjects had poor self-perceived health status, seeking social support coping was high $(\mathrm{p}<0.05)$. If they were dissatisfied with their economic level, problem-focused coping and seeking social support coping were the highest, whereas if satisfied with their economic level, wishful thinking coping was high $(\mathrm{p}<0.05)$. As the subjects were more dissatisfied with campus life and the dental hygiene major, problem-focused coping and seeking social support coping were higher, showing statistically significant differences $(p<0.05)$.

\section{Correlation between empathic ability, campus life stress and stress coping behaviors}

The results of examining the correlation between empathic ability, campus life stress, and stress coping behaviors are shown in Table 3 . With regard to the correlation between empathic ability and the subdomains of campus life stress, interpersonal relationship stress had a low negative correlation with empathic concern and a low positive correlation with personal distress $(\mathrm{p}<0.01)$. Task-related stress was correlated with personal distress $(\mathrm{r}=0.361, \mathrm{p}<0.001)$. With regard to the correlation between emphatic ability and the subdomains of stress coping behavior, the highest correlation was between personal distress and wishful thinking coping with 0.328 $(\mathrm{p}<0.001)$, followed by the correlation between viewpoint acceptance and problem-focused coping $(r=0.240, p$ $<0.001$ ), and the correlation between emphatic concern and seeking social support coping $(\mathrm{r}=0.237, \mathrm{p}<0.001)$. With regard to the relationship between campus life stress and the subdomains of stress coping behavior, there was the highest correlation between task-related stress and wishful thinking coping with $0.306(\mathrm{p}<0.001)$.

\section{Factors affecting stress coping behaviors}

Factors affecting problem-focused coping among the

Table 3. Relationship of Empathic Ability, Campus Life Stress and Stress Coping Behaviors $(n=395)$

\begin{tabular}{|c|c|c|c|c|c|c|c|c|c|c|}
\hline & $\mathrm{PT}$ & FS & $\mathrm{EC}$ & PD & IRS & TRS & PFC & SSS & SEF & WT \\
\hline PT & 1 & & & & & & & & & \\
\hline FS & $0.159 * *$ & 1 & & & & & & & & \\
\hline EC & $0.272 * * *$ & $432 * * *$ & 1 & & & & & & & \\
\hline PD & $0.154 * * *$ & $237 * * *$ & $0.306 * * *$ & 1 & & & & & & \\
\hline IRS & -0.046 & -0.019 & $-0.135^{* *}$ & $0.161 * *$ & 1 & & & & & \\
\hline TRS & 0.075 & 0.033 & -0.048 & $0.361 * * *$ & $0.512 * * *$ & 1 & & & & \\
\hline PFC & $0.240 * * *$ & $0.100^{*}$ & 0.044 & -0.039 & 0.007 & -0.042 & 1 & & & \\
\hline SSS & $0.235^{* * *}$ & $0.203 * * *$ & $0.237 * * *$ & $0.140 * *$ & -0.004 & 0.008 & $0.481 * * *$ & 1 & & \\
\hline SEF & 0.065 & -0.063 & -0.081 & 0.095 & 0.085 & $0.113^{*}$ & $0.191 * * *$ & $0.126^{*}$ & 1 & \\
\hline WT & $0.158 * *$ & $0.230 * * *$ & $0.112 *$ & $0.328 * * *$ & 0.047 & $0.306^{* * *}$ & $0.225 * * *$ & $0.364 * * *$ & $0.297 * * *$ & 1 \\
\hline
\end{tabular}

PT: perspective-taking, FS: fantasy scale, EC: empathic concern, PD: personal distress, IRS: interpersonal relationship stress, TRS: task-related stress, PFC: problem focus coping, SSS: seeking social support, SEF: support emotional focus, WT: wishful thinking. ${ }^{*} \mathrm{p}<0.05, * * \mathrm{p}<0.01, * * * \mathrm{p}<0.001$ 
Table 4. Variables Affecting Stress Coping Behaviors of Subjects $(n=395)$

\begin{tabular}{|c|c|c|c|c|c|}
\hline Variable & $\mathrm{B}$ & SE & $\beta$ & $\mathrm{t}$ & $\mathrm{p}$ \\
\hline \multicolumn{6}{|l|}{ Problem focus coping } \\
\hline Perspective-taking & 0.327 & 0.067 & 0.240 & 4.894 & $<0.001$ \\
\hline \multicolumn{6}{|c|}{$\mathrm{F}=23.952, \mathrm{R}^{2}=0.240$, adjusted $\mathrm{R}^{2}=0.057, \mathrm{p}<0.001$} \\
\hline \multicolumn{6}{|l|}{ Seeking social support } \\
\hline Empathic concern & 0.180 & 0.071 & 0.139 & 2.535 & 0.012 \\
\hline Perspective-taking & 0.263 & 0.073 & 0.179 & 3.587 & $<0.001$ \\
\hline Fantasy scale & 0.120 & 0.056 & 0.115 & 2.159 & 0.031 \\
\hline \multicolumn{6}{|c|}{$\mathrm{F}=14.234, \mathrm{R}^{2}=0.098$, adjusted $\mathrm{R}^{2}=0.092, \mathrm{p}<0.001$} \\
\hline \multicolumn{6}{|l|}{ Support emotional focus } \\
\hline Task-related stress & 0.106 & 0.047 & 0.113 & 2.254 & 0.025 \\
\hline \multicolumn{6}{|c|}{$F=5.082, R^{2}=0.013$, adjusted $R^{2}=0.010, p=0.025$} \\
\hline \multicolumn{6}{|l|}{ Wishful thinking } \\
\hline Personal distress & 0.255 & 0.063 & 0.203 & 4.023 & $<0.001$ \\
\hline Task-related stress & 0.280 & 0.053 & 0.295 & 5.255 & $<0.001$ \\
\hline Fantasy scale & 0.159 & 0.044 & 0.170 & 3.613 & $<0.001$ \\
\hline Interpersonal relationship stress & -0.143 & 0.057 & -0.134 & -2.521 & 0.012 \\
\hline \multicolumn{6}{|c|}{$\mathrm{F}=22.818, \mathrm{R}^{2}=0.190$, adjusted $\mathrm{R}^{2}=0.181, \mathrm{p}<0.001$} \\
\hline
\end{tabular}

The data was analysed by the stepwise multiple regression analysis.

SE: standard error.

subdomains of stress coping behavior were view acceptance among the subdomains of emphatic ability $(p<$ 0.001). Seeking social support coping was affected by emphatic concern, viewpoint acceptance and fantasy factors $(\mathrm{p}<0.001)$. A higher level of task-related stress as a subdomain of campus life stress had higher impact on emotional relief coping $(p=0.025)$. Factors affecting wishful thinking coping included personal distress, task-related stress, fantasy, and interpersonal relationship stress, these were statistically significant ( $p<0.001$, Table 4$)$.

\section{Discussion}

The present study aimed to investigate the effects of empathic ability and campus life stress on stress coping behaviors in dental hygiene students.

The score for empathic concern among the subdomains of empathic ability was the highest with 3.60 points, whereas the score for viewpoint acceptance was the lowest with 3.19 points. These results were generally low when compared with the results of a study by Jeong and Lee ${ }^{15)}$ conducted on nursing students using the same instrument. In that study, the score for personal distress was found to be the lowest with 3.15 points, which was different from the results of the present study. The most important factor for dental hygiene students, as future dental hygienists, is empathic ability in order to understand the others' suffering and provide good quality dental care service. In order to improve their empathic ability, various programs should be developed and education, provided to enhance their interaction and communication skills, should be easily accessible. In terms of campus life stress, the score for task-related stress (2.72 points) was higher than the score for interpersonal relationship stress (1.65 points).

This finding was consistent with the results of a study of nursing students by Park $^{16)}$ showing that the score for stress coping behavior measured on a 4-point scale was only somewhat lower than the results of the present study. The reason is assumed to be that both dental hygiene and nursing students were stressed due to academic grade management and adaptation to clinical practice as they participated in academic studies and clinical practice concurrently. In addition, because they can be employed after passing a national examination for obtaining a license, they are thought to experience a higher level of task-related stress than that of interpersonal relationship stress. This seems to be a reflection of the characteristics of health science majors. Various studies on social, 
psychological, and environmental factors affecting students' stress and stress coping are needed.

As for the differences in stress coping behaviors according to the general characteristics of the subjects, the lower age and academic year, the higher problem-focused coping. If the subjects were religious, seeking social support coping was found to be high. A previous study of health science students by $\mathrm{Yu}^{17)}$ revealed that problem-focused coping was higher in the subjects in a higher academic year; this conflicts with the results of the present study. However, that study reported that seeking social support coping was high in those who were religious, which was consistent with the present study. The results of this present study found that by sex, both emotional relief coping and wishful thinking coping were higher in men than women, which conflicts with the results of the study by $\mathrm{Yu}^{17)}$. According to the results of a previous study, problem-focused coping and seeking social support coping were found to be effective in relieving stress and led to feelings of happiness, but emotional relief coping and wishful thinking coping were found to be ineffective in solving problems or relieving stressors, and to reduce a feeling of happiness ${ }^{18)}$. Based on these results, it was found that if the subjects were female, lower in age and academic year, and were religious, their stress coping behaviors were more effective and efficient, they chose active stress coping behaviors, and made efforts to cope with stress. In order to improve the overall coping ability and the stress coping abilities in dental hygiene students in the future, it is thought that the college educational environment should be changed with a focus on the improvement of problem-solving ability and seeking social support in dental hygiene students.

In the present study, there was a low negative correlation between empathic concern among emphatic ability and interpersonal relationship stress among campus life stress $(p<0.01)$, whereas there was a positive correlation between personal distress among emphatic ability and task-related stress among campus life stress $(\mathrm{r}=0.361, \mathrm{p}<0.001)$. A study of college students by Jang $^{19)}$ showed a low negative correlation between empathic concern and stress level, and the highest positive correlation between personal distress and stress level.
These findings were consistent with the results of the present study. The ability to understand and accept the other party's feelings or situations can be interpreted as having a low level of interpersonal stress because the ability is linked to smooth interpersonal relationships. It can be evident that dental hygiene students working in clinical practice while seeing patients' suffering and pain experienced increased task-related stress in the face of personal distress. This supports the results of previous studies $^{20,21)}$ showing that emotional intelligence was related to stress, and also suggests that the ability to control emotional intelligence is needed. As for the relationship between emphatic ability and the stress coping behavior subdomains, those with higher viewpoint acceptance ability and empathic concern used active coping strategies such as task-related coping and seeking social support coping, and those having higher fantasy and personal distress were more likely to choose wishful thinking coping behaviors. In terms of the relationship between campus life stress and stress coping behavior, those with a higher level of task-related stress were more likely to choose wishful thinking coping. In order to more positively accept and proactively cope with stress in campus and daily life, it is thought that measures should be provided to improve viewpoint acceptance and empathic concern ability, and related college curricula should be established.

It was found that the factor affecting both problem-focused coping and seeking social support coping among the subdomains of stress coping behavior was viewpoint acceptance, while the factor affecting both emotional relief coping and wishful thinking coping was task-related stress. Stress cannot be avoided in our life. Although the types of stress individuals experience are important, it is more important to cope with and deal with the stress. Expert advice and guidance will be necessary to improve the quality of life and happiness index in dental hygiene students by helping them more wisely cope with the complex stress that they experience due to clinical practice, the national examination, and employment. As future hygienists, dental hygiene students will have jobs that require empathy with patients. In view of the fact that job stress is directly related to work efficiency, it is 
thought that an educational program should be provided to help them cope with stress and build trusting relationships through interactions with others.

Because the subjects of the present study were selected by convenience sampling among dental hygiene students at 3 colleges located in Gyeonggi-do, Chungcheongnam-do and Daejeon City, South Korea, it is difficult to generalize the results of this study. In addition, in view of the fact that there was a big difference in the number of male and female subjects, it is difficult to compare gender differences. Nevertheless, this present study is significant in that it investigated emphatic ability, campus life stress, and stress coping behavior in dental hygiene students by dividing these findings into subdomains and identifying factors affecting stress coping behaviors. To adjust for these limitations more comprehensive and multi-faceted studies should be conducted in the future.

\section{References}

1. Davis MH: A multidimensional approach to individual differences in empathy. JSAS Cat Sel Doc Psychol 10: 85, 1980.

2. Daum: Stress. Retrieved July 2, 2017, from http://dic. daum.net/word/view.do? wordid=kkw000154638\&;supid= kku000195325(2009, October 9).

3. Lee CH, Kim JH: Relations of perceived stress, cognitive set, and coping behaviors to depressidn: a focus on freshmen's stress experiences. Korean J Counsel Psychother 1: 25-45, 1988.

4. Kim KH, Chon KK: The effects of psychological stress and coping in college students. Korean J Stress Res 2: 229-237, 1994.

5. Richard SL, Folkman S, Kim JH: Stress, appraisal, and coping. 1st ed. Daekwang, Seoul, pp.173-178, 2001.

6. Choi HS: All human emotions. 1st ed. Seohaemoonjib, Paju, p.246, 2011.

7. Kim MH: A study on ego-identify, interpersonal relations ability, and adjustment in nursing students. J Korean Acad Psychiatr Ment Health Nurs 17: 55-64, 2008.

8. Park JH, Chung OB: The family system types of circumplex model and ego identity development in adolescence. Fam
Environ Res 40: 49-63, 2002.

9. Gong SJ, Lee EH: Mediation effect of coping between life stress and depression in female college students. Korean J Woman Psychol 11: 21-40, 2006.

10. Choi MK: Symptoms, depression, and coping behaviors of university students. Taehan Kanho Hakhoe Chi 33: 433-439, 2003.

11. Kang I, Kee S, Kim SE, et al.: Reliability and validity of the Korean-version of interpersonal reactivity index. J Korean Neuropsychiatr Assoc 48: 352-358, 2009.

12. Chon KK, Kim KH, Yi JS: Development of the revised life stress scale for college students. Korean J Health Psychol 5: 316-355, 2000.

13. Kim JH: Relations of perceived stress, cognitive set, and coping behaviors to depression. Unpublished doctoral dissertation, Seoul National University, Seoul, 1987.

14. Folkman S, Lazarus RS: If it changes it must be a process: study of emotion and coping during three stages of a college examination. J Pers Soc Psychol 48: 150-170, 1985.

15. Jeong HS, Lee KL: Empathy and clinical practice stress in nursing student. J Korea Acad-Ind Coop Soc 16: 6044-6052, 2015.

16. Park PN: The mediating effect of stress coping type and self-esteem between life stress and suicidal ideation in nursing college students. J Korean Soc Sch Health 29: 1-10, 2016.

17. Yu EY: Self-esteem and stress-coping strategies of the college students in the department of public health. J Korea Contents Assoc 9: 225-236, 2009.

18. Monat A, Lazarus RS: Stress and coping: an anthology. 1st ed. Columbia University Press, New York, pp.207-227, 1991.

19. Jang HS: The effects of self-disclosure and empathy on stress perception and stress coping strategy of university students. Korean J Commun Stud 21: 5-28, 2013.

20. Cho HC: Relations between gifted students' emotional intelligence and their social skills, school adjustment, stress and stress coping strategies. J Korean Soc Gift Talent 9: 121-140. 2010.

21. Song JE, Chae HJ: Differences in stress and stress coping strategies according to emotional intelligence in nursing students. J Korean Acad Soc Nurs Educ 20: 502-512, 2014. 